\title{
CLS Data Interpolation with Spline Curves and Its Post Processing for Generating a Robot Language
}

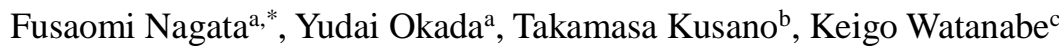 \\ aDepartment of Mechanical Engineering, Faculty of Engineering, Tokyo University of Science, Yamaguchi \\ Sanyo-Onoda 756-0884, Japan \\ bSOLIC Co., Ltd. \\ Omuta, 836-0064, Japan \\ ${ }^{\mathrm{c}}$ Graduate School of Natural Science and Technology, Okayama University \\ Okayama 700-8530, Japan \\ *Corresponding Author: nagata@rs.tusy.ac.jp
}

\begin{abstract}
In this paper, an interpolation method with spline curves is proposed to smooth cutter location source data called CLS data. $3^{\text {rd }}$-order and $5^{\text {th }}$-order spline curves are easily implemented for interpolation. Then, a post processor is designed for an industrial robot FANUC R2000iC to produce FANUC robotic program called LS format. The post processor works as a straightforward and cooperative interface between CAD/CAM systems and the industrial robot, so that a desirable teaching-less operation environment is realized. The validity and usefulness of the proposed methods are evaluated through a simulation on FANUC RoboGuide and an actual machining experiment with the robot. The FANUC RoboGuide is a robotic simulation environment that can execute both the robot's motion and application, largely reducing the setup time and risk in using an actual robot.
\end{abstract}

Keywords: Industrial robot, interpolation, spline curve, CAD/CAM, CLS data, Teaching less, FANUC robotic program, LS format, Machining robot.

\section{Introduction}

Up to now, a lot of researches and efforts have been dedicated to develop user-friendly data interface for industrial robots. For example, Xuejuan et al. presented a novel robot sculpturing planning method for the 3D sculpturing with CAD-geometry data. An automatic trajectory generation from the $3 \mathrm{D}$ CAD models of freeform surfaces to the machining tool path by the linear interpolation was described [1]. Solvang et al. considered a robotic CAM for machining operations using an industrial robot. The system coped with the new machining standard codes called STEP-NC [2], which is a machine tool control language including geometric dimension and tolerance data for inspection. Also, many off-line programming methods have been proposed to reduce the load of robot programming. However, some practical issues such as cable/hose tangling, robot configuration, collision and reachability have not been well overcome yet. Chen and Sheng discussed a new method to generate robot programs while dealing with those problems [3].

The authors presented a machining system based on an industrial robot RV1A for foamed polystyrene materials [4]. The direct servo system developed for the robot could deal with NC and CLS files as desired trajectories, so that a useful interface without using a robot language between operators and the machining robot could be realized. The CLS means cutter location source which is a standardized tool path generated by the main processor of CAM. Further, a 3D printer-like data interface called the preprocessor has been proposed for industrial robots [5]. In the interface, triangulated patch based path, zigzag or spiral one can be smartly produced from STL data without conducting a complicated teaching task and using a commercially provided CAD/CAM system. STL means the Stereolithography which was proposed by 3D Systems and is widely used by additive layered manufacturing systems such as $3 \mathrm{D}$ printers. 
What we propose in this paper is two software. The first one is an interpolation method with spline curves which smooths CLS data. $3^{\text {rd }}$-order and $5^{\text {th }}$-order spline curves are easily implemented with a simple linear algebra for interpolation. The second one is a post processor for an industrial robot FANUC R2000iC to realize a complete teaching-less operation environment. An early concept of robotic post processor is described in [6]. On the other hand, the post-processor proposed in this paper can directly generate rigorous and complex FANUC robotic programs from CLS data. The program is a human-readable ASCII file and is call LS data. As is well-known, the CLS data are made by using ordinary CAD/CAM systems. The proposed postprocessor works as a straightforward and cooperative interface between CAD/CAM systems and the industrial robot. The convenience and usefulness of the proposed postprocessor are evaluated through a simulation on FANUC RoboGuide and an actual machining experiment with the robot. The FANUC RoboGuide is a robotic simulation environment that can execute both the robot's motion and application, largely reducing the setup time and risk in using an actual robot.

\section{CLS Data Interpolation with Spline Curves}

When the target model has some curved surface, the main processor of CAD/CAM generally constructs the surface, i.e., CLS data, with minute straight lines by a linear approximation. CLS data interpolation is effective to smooth such a linear approximated trajectory.

\subsection{In case of $3^{\text {rd }}$-order spline curve}

Figure 1 shows three $3^{\text {rd }}$-order spline curve $x_{i-1(t)}$, $\boldsymbol{x}_{i(t)}$ and $\boldsymbol{x}_{i+1(t)}$ just passing on four points $\boldsymbol{p}_{i-1}, \boldsymbol{p}_{i}$, $\boldsymbol{p}_{i+1}$ and $\boldsymbol{p}_{i+2}\left(\in \mathfrak{R}^{3 \times 1}\right)$. The spline curve $\boldsymbol{x}_{i}(t)$ is written as

$$
\boldsymbol{x}_{i}(t)=\boldsymbol{a}_{i} t^{3}+\boldsymbol{b}_{i} t^{2}+\boldsymbol{c}_{i} t+\boldsymbol{d}_{i}
$$

Where $\boldsymbol{x}_{i}(t)=\left[x_{i}(t) y_{i}(t) z_{i}(t)\right]^{T}$ is the spline curve in Cartesian space; $\boldsymbol{a}_{i}(t)=\left[a_{x i}(t) a_{y i}(t) a_{z i}(t)\right]^{T}, \boldsymbol{b}_{i}(t)=$ $\left[b_{x i}(t) b_{y i}(t) b_{z i}(t)\right]^{T}, \quad \boldsymbol{c}_{i}(t)=\left[c_{x i}(t) c_{y i}(t) c_{z i}(t)\right]^{T}$ and $\boldsymbol{d}_{i}(t)=\left[d_{x i}(t) d_{y i}(t) d_{z i}(t)\right]^{T}$ are the coefficients vectors; $t$ is the normalized variable. When the $x$-component is considered, the following relations are obtained from Fig. 1.

$$
x_{i}(-1)=p_{x(i-1)}=-a_{x i}+b_{x i}+c_{x i}+d_{x i}
$$

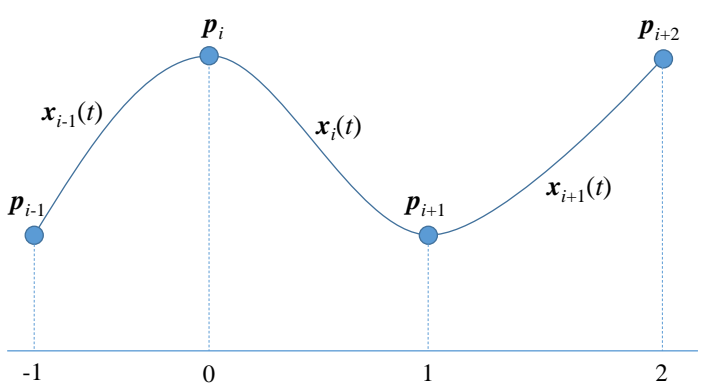

Fig. 1. $3^{\text {rd }}$-order spline curves for CLS data interpolation.

$$
\begin{array}{r}
x_{i}(0)=p_{x i}=d_{x i} \\
x_{i}(1)=p_{x(i+1)}=a_{x i}+b_{x i}+c_{x i}+d_{x i} \\
x_{i}(2)=p_{x(i+2)}=8 a_{x i}+4 b_{x i}+2 c_{x i}+d_{x i}
\end{array}
$$

$d_{x i}$ is fixed as $p_{x i}$, so that the remaining three coefficients are calculated by

$$
\left(\begin{array}{l}
a_{x i} \\
b_{x i} \\
c_{x i}
\end{array}\right)=\left(\begin{array}{ccc}
-\frac{1}{6} & \frac{1}{2} & -\frac{1}{6} \\
-\frac{1}{2} & \frac{1}{2} & 0 \\
\frac{1}{3} & 1 & -\frac{1}{6}
\end{array}\right)\left(\begin{array}{c}
p_{x(i-1)}-p_{x i} \\
p_{(i+1)}-p_{x i} \\
p_{x(i+2)}-p_{x i}
\end{array}\right)
$$

Coefficients in other two directions are similarly obtained, so that the section between $\boldsymbol{p}_{i}$ and $\boldsymbol{p}_{i+1}$ can be interpolated with $x_{i}(t)(0 \leq t \leq 1)$. By applying this procedure to all spline curves, the original CLS data can be interpolated for smoothing.

\subsection{In case of $5^{\text {th }}$-order spline curve}

Next, fifth-order spline curve is also considered to interpolate CLS data. Figure 2 shows three $5^{\text {th }}$-order spline curves $x_{i-2}(t), x_{i-1}(t), x_{i}(t), x_{i+1}(t)$ and $x_{i+2}(t)$ just passing on six points $\boldsymbol{p}_{i-2}, \boldsymbol{p}_{i-1}, \boldsymbol{p}_{i}, \boldsymbol{p}_{i+1}, \boldsymbol{p}_{i+2}$ and $\quad \boldsymbol{p}_{i+3}\left(\in \mathfrak{R}^{3 \times 1}\right)$. The spline curve $\boldsymbol{x}_{i}(t)=\left[\begin{array}{lll}x_{i}(t) & y_{i}(t) & z_{i}(t)\end{array}\right]^{T}$ is written as

$$
\boldsymbol{x}_{i}(t)=\boldsymbol{a}_{i} t^{5}+\boldsymbol{b}_{i} t^{4}+\boldsymbol{c}_{i} t^{3}+\boldsymbol{d}_{i} t^{2}+\boldsymbol{e}_{i} t+\boldsymbol{f}_{i}
$$

In the same manner as the case of $3^{\text {rd }}$-order spline curves, when $x$-directional component is considered, the following relations are obtained from Fig. 2.

$$
x_{i}(-2)=p_{x(i-2)}=
$$




$$
\begin{array}{r}
-32 a_{x i}+16 b_{x i}-8 c_{x i}+4 d_{x i}-2 e_{x i}+f_{x i} \\
x_{i}(-1)=p_{x(i-1)}=-a_{x i}+b_{x i}-c_{x i}+d_{x i}-e_{x i}+f_{x i} \\
x_{i}(0)=p_{x i}=f_{x i} \\
x_{i}(1)=p_{x(i+1)}=a_{x i}+b_{x i}+c_{x i}+d_{x i}+e_{x i}+f_{x i} \\
x_{i}(2)=p_{x(i+2)}= \\
32 a_{x i}+16 b_{x i}+8 c_{x i}+4 d_{x i}+2 e_{x i}+f_{x i} \\
x_{i}(3)=p_{x(i+3)}= \\
243 a_{x i}+81 b_{x i}+27 c_{x i}+9 d_{x i}+3 e_{x i}+f_{x i}
\end{array}
$$

which leads to

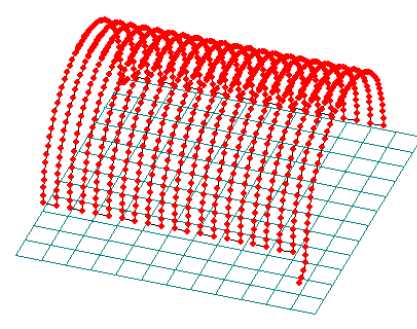

Original CLS data

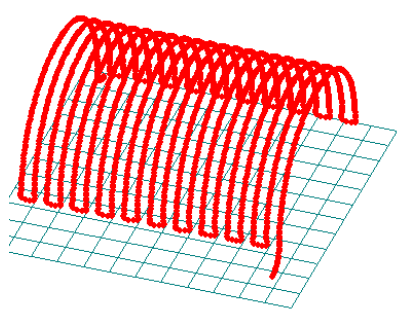

Interpolated CLS data

Fig. 3. Example of original CLS data and interpolated ones with $3^{\text {rd }}$-order spline curves.
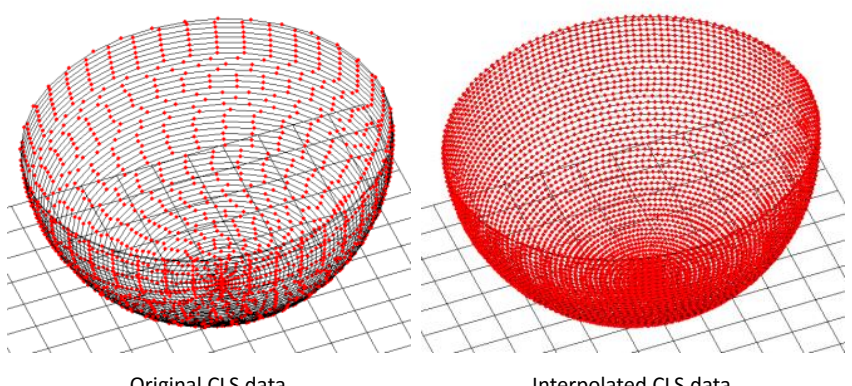

Interpolated CLS data

Fig. 4. Comparison of original CLS data and interpolated ones with $5^{\text {th }}$-order spline curves.

\section{Post Processing}

that the section between $\boldsymbol{p}_{i}$ and $\boldsymbol{p}_{i+1}$ can be interpolated with $\boldsymbol{x}_{i}(t)(0 \leq t \leq 1)$.

An experiment of interpolation of a spiral path was conducted with $3^{\text {rd }}$ - and $5^{\text {th }}$-order spline curves. The left side of Fig. 3 shows the points in original CLS data. Also, the right side figure draws the points in the CLS data interpolated with $3^{\text {rd }}$-order spline curves. The number of interpolated points within a section is set to three. Figure 4 shows another example of $5^{\text {th }}$-order spline curves. As can be seen, the interpolations are well applied to the original CLS data for smoothing.

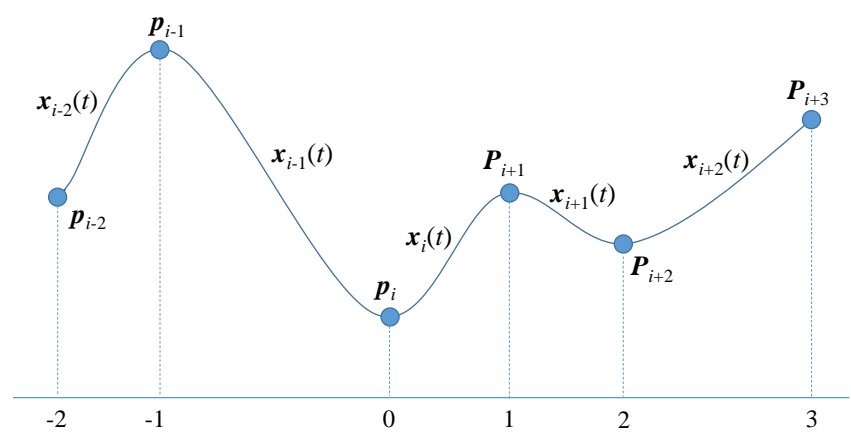

Fig. 2. $5^{\text {th }}$-order spline curves for CLS data interpolation.

In this section, a post processor is introduced for an industrial robot FANUC R2000iC to produce a robot program written with LS format from CLS data. Figure 5(a) is the past proposed process 1 , in which the machining robot RV1A could work based on CLS data generated from the main processor of CAD/CAM, without conducting any teaching tasks and using robot languages [4].

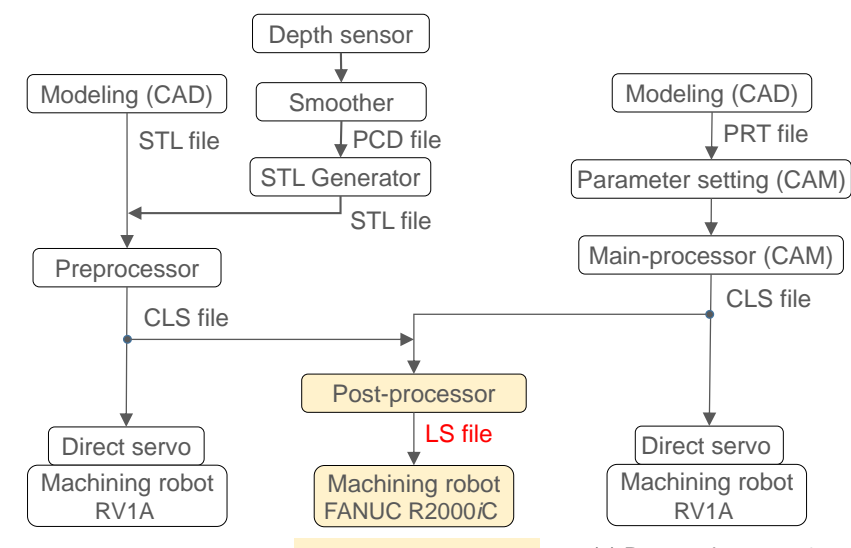

(b) Proposed process 2

(c) Proposed process 3

(a) Proposed process 1

Fig. 5. Flowcharts of the past developed processes and newly proposed post processor for FANUC R2000iC. 
Figure 5(b) is the past proposed process 2, in which the preprocessor could produce zigzag-based and spiral-based CLS data from STL data without using commerciallyprovided CAD/CAM systems [5]. Whereas the post processor proposed in this paper is illustrated in the flow chart of Fig. 5(c). The post processor generates FUNUC robotic program called LS data from CLS data, so that desirable operation environment as in using $\mathrm{NC}$ machine tools can be realized for the robot. As is well-known, NC machine tools are controlled based on NC data postprocessed from CLS data.

\section{About Robotic Program of FANUC R2000iC Series}

Figure 6 illustrates the ASCII file format of FANUC robotic program whose file extension is given by ".LS". A LS file is composed of three areas: header, program and data areas. First of all, the header area consists of the following statements.
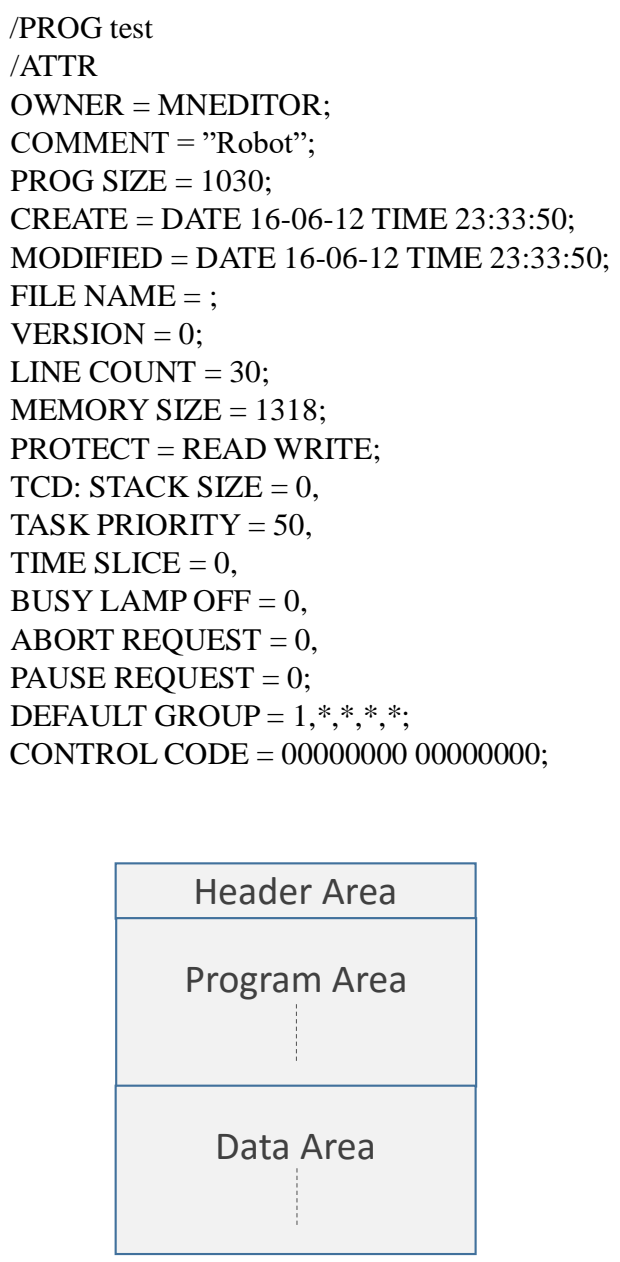

Fig. 6. ASCII file format of FANUC robotic program called LS data.
The number of lines in the header area is fixed to 20. For example, at the line of "PROG", the file name of LS file (test.ls) must be written as "test". At the line of "LINE COUNT =", the sum of number of "JP" and "LP" in program area must be written. "JP" and "LP" have similar meanings as "G00" and "G01" in NC program, respectively. Then, the program area with variable number of lines is written as the following commands.

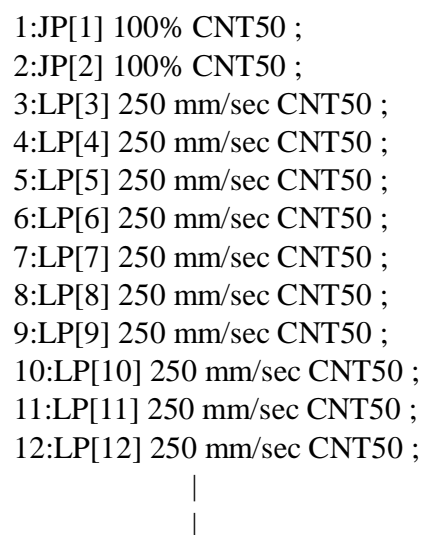

where "JP" means rapid motion like "G00" in NC program. "JP" is effective to rapidly manuever an end mill to the position near the initial cut for removing material. "LP" means feed motion with a specified feed rate $[\mathrm{mm} / \mathrm{sec}]$ like "G01". "CNT50" is added to round the corner off with a radius $50 \mathrm{~mm}$.

Finally, the data area is written as mainly the following position and orientation components in Cartesian space. The size of the data area is also variable according to teaching points for playback motion.

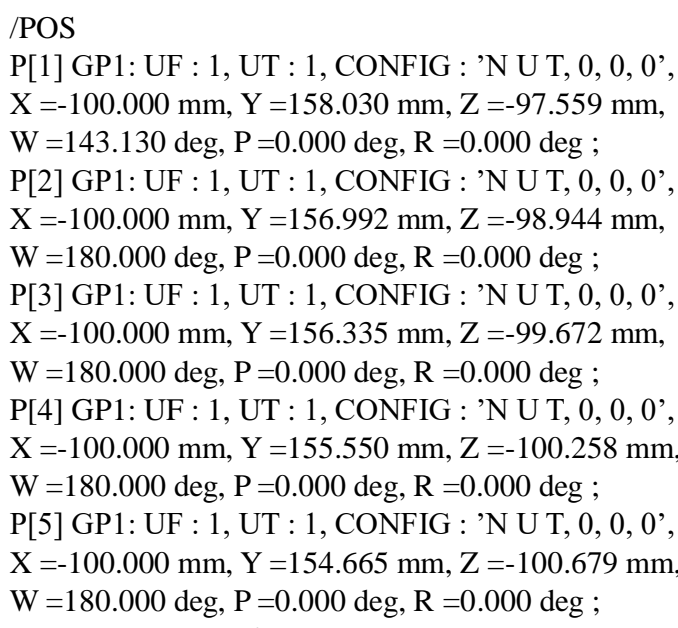

where $\mathrm{X}, \mathrm{Y}$ and $\mathrm{Z}$ are the components of a position vector; $\mathrm{W}, \mathrm{P}$ and $\mathrm{R}$ mean the rotational angles around $x$-, $y$ - and $z$ - 
axes, respectively. "UF" and "UT" are the parameters for work and tool coordinate systems, respectively. The work and tool coordinate systems should be set in advance. "CONFIG" is for the description of configuration of the industrial robot actually used. In the next section, the proposed post processor smartly produces the above explained FANUC robotic program called LS data from CLS data outputted from the main process of a CAD/CAM.

\section{Post Processor to Produce FANUC Robotic Program from CLS Data}

The proposed post processor yields FANUC robot program (.LS) from CLS data. As an example, sample CLS data viewed as Fig. 7 are listed as

GOTO/0.0000,-94.3881,39.3232,0.0000000,0.2545675,0.9670550 GOTO/0.0000,-98.2856,40.3157,0.0000000,0.2389777,0.9710251 GOTO/0.0000,-102.1985,41.2453,0.0000000,0.2233261,0.9747438 GOTO/0.0000,-106.1258,42.1120,0.0000000,0.2076166,0.9782103 GOTO/0.0000,-110.0666,42.9153,0.0000000,0.1918535,0.9814236 GOTO/0.0000,-114.0198,43.6551,0.0000000,0.1760406,0.9843829

Where each "GOTO" statement in CLS file has a position vector $\boldsymbol{x}=\left[\begin{array}{lll}x & y & z\end{array}\right]^{T}$ and a normal vector $\boldsymbol{n}=\left[\begin{array}{lll}n_{x} & n_{y} & n_{z}\end{array}\right]^{T}$. Thus, the rotation angles $w, p, r$ [deg.] in LS file need to be calculated from the components of $\boldsymbol{n}$. In the case of FANUC R2000iC, $w$ and $p$ are obtained by

$$
\begin{aligned}
& w=-\arctan 2\left(-n_{y},-n_{z}\right) \\
& p=\arcsin \left(n_{x}\right)
\end{aligned}
$$

where $\arctan 2$ and $\arcsin$ are inverse trigonometric functions; $\arctan 2$ generates the angle within the range from $-\pi$ to $\pi$. It should be noted that the angle $r$ can be fixedly set to 0 because of the axis-symmetry of a motor spindle attached to the arm tip.

An experiment of conversion from CLS data shown in Fig. 7 to FANUC LS data was conducted. Then, the validity of the converted, i.e., post-processed LS data was examined through a playback experiment on a simulation environment RoboGuide provided by FANUC. Figure 8 shows the simulation result using the model of FANUC R2000iC, in which an actual trajectory of the tip of the ball-end mill is traced. If the post-processed LS data contain some inconsistency from the viewpoint of the format of FANUC robotic program language, then the RoboGuide warns the format error. However, it was confirmed from the simulation that the tip of the ball-end mill could successfully follow the zigzag-based path without any warning as shown in Fig. 8.

Further, a machining experiment using an actual industrial robot FANUC R2000iC was carried out as shown in Fig. 9 by giving FANUC robot program (LS data) postprocessed from another CLS data, so that the foamed polystyrene material could be successfully formed into the designed shape.

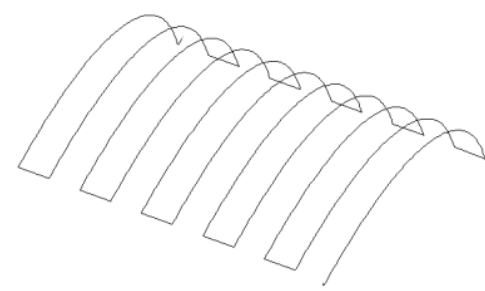

Fig. 7. A part of CLS data (zigzag path) generated from a freeformed surface designed by CAD/CAM Creo.

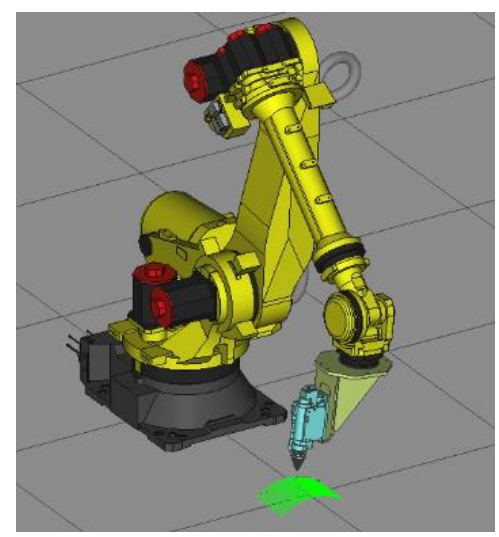

Fig. 8. Successful playback experiment using LS data postprocessed from CLS data, i.e., trajectory following control of the tip of the ball-end mill.

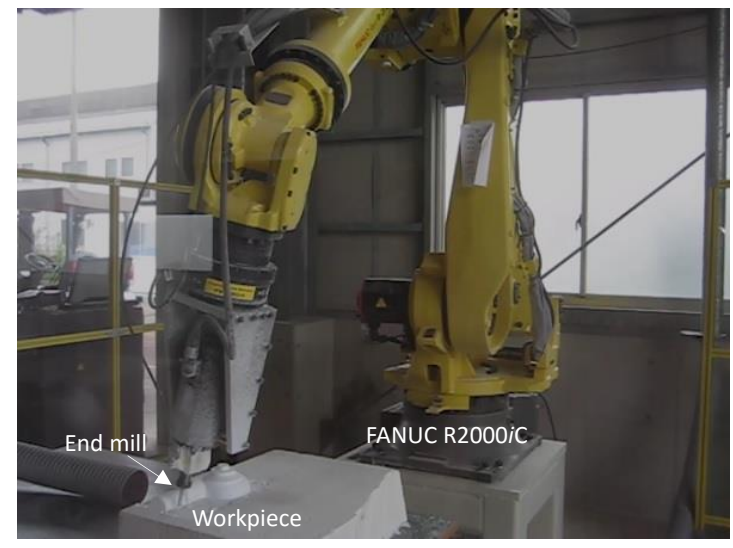

Fig. 9. Machining scene of FANUC R2000iC dealing with FANUC robot program post-processed from CLS data (SOLIC Co., Ltd., Omuta, Japan). 


\section{Conclusions}

In this paper, an interpolation method with spline curves has been first proposed to smooth original cutter location source data called CLS data. $3^{\text {rd }}$-order and $5^{\text {th }}$-order spline curves were easily implemented with a simple linear algebra for interpolation. Then, a post processor for an industrial robot FANUC R2000iC has been introduced. Without conventional teaching process, the post processor could regularly and consistently generate FANUC robotic program called LS data from cutter location source data called CLS data. CLS data are generally made through the main process of $\mathrm{CAD} / \mathrm{CAM}$, so that a promising data interface between the industrial robot and $\mathrm{CAD} / \mathrm{CAM}$ could be designed for realizing a teaching-less operation environment. The effectiveness and usefulness were confirmed through a playback experiment on a simulation environment called RoboGuide and an actual machining experiment.

It is also another advantage that CLS data can be converted from STL data by using the already developed preprocessor [5] and then LS data can be further made from the CLS data using the proposed post processor. This means that FANUC robotic program can be consequently constructed from STL data used with a de facto standard for $3 \mathrm{D}$ printers.

\section{Acknowledgment}

This work was supported by JSPS KAKENHI Grant Number JP16K06203.

\section{References}

[1] N. Xuejuan, L.Jingtai, S. Lei, L. Zheng and C. Xinwei : "Robot 3D sculpturing based on extracted NURBS", Procs. of IEEE International Conference on Robotics and Biomimetics (ROBIO2007), pp. 1936-1941, 2007.

[2] B. Solvang, L.K. Refsahl and G. Sziebig : "STEP-NC based industrial robot CAM system", Procs. of the 9th International Symposium on Robot Control (SYROCO'09), pp. 361-366, 2009.

[3] H. Chen and W. Sheng : "Transformative CAD based industrial robot program generation", Robotics and Computer-Integrated Manufacturing, vol. 27, no. 5, pp. 942948, 2011.

[4] F. Nagata, A. Otsuka, K. Watanabe and M.K. Habib : "Machining robot for foamed polystyrene materials using fuzzy feed rate controller", International Journal of
Mechatronics and Automation, vol. 5, no. 1, pp. 34-43, 2015. [5] F. Nagata, K. Takeshita, K. Watanabe and M.K. Habib : "Generation of triangulated patches smoothed from original point cloud data with noise and its application to robotic machining", Procs. of The 2016 IEEE International Conference on Mechatronics and Automation (ICMA 2016), pp. 535-540, 2016.

[6] S.G. Andrews: Developments in post-processing for offline robot programming, Advances in Manufacturing Technology II, pp 165-169, 1987. 